\title{
Raman Amplification of Charge-15 Orbital Angular Momentum Mode in a Large Core Step Index Fiber
}

\author{
Sheng Zhu ${ }^{1 *}$, Srinivas Pachava ${ }^{2}$, Shankar Pidishety ${ }^{1}$, Yutong Feng ${ }^{1}$, Balaji Srinivasan ${ }^{2}$ and Johan Nilsson ${ }^{1}$ \\ ${ }^{1}$ Optoelectronics Research Centre, University of Southampton, Southampton SO17 1BJ, UK \\ ${ }^{2}$ Department of Electrical Engineering, Indian Institute of Technology Madras, Chennai-600036, India \\ *Email: Zhu.S@soton.ac.uk
}

\begin{abstract}
We report 9.2-dB Raman amplification of pulses at $1121 \mathrm{~nm}$ in an orbital angular momentum mode with charge 15 in $30 \mathrm{~m}$ of $50-\mu \mathrm{m}$-diameter step-index-core fiber. The amplified signal mode-purity is measured to $78.4 \%$.

OCIS codes: (060.2320) Fiber optics amplifier and oscillators, (060.4370) Nonlinear optics, fibers
\end{abstract}

Fiber Raman amplifiers (FRAs) have been demonstrated to be a viable avenue for amplification of higher order modes (HOMs), including orbital angular momentum modes (OAMMs), in multimode step-index fibers [1,2], with good preservation of the mode purity. In this regard, non-local gain saturation and gain media with easy refractive index control are attractive features of FRAs. For OAMMs, it is also known that the coupling to the conjugate mode is less likely for high charge $(l>10)$ [3], which points to the possibility of better purity preservation, e.g., compared to what we achieved for an $l=2$ OAMM [2]. Here, we present Raman amplification of a first radial order OAMM with azimuthal order (i.e., charge) $l=15$ in a $30-\mathrm{m}$ fiber with a $50-\mu \mathrm{m}$-diameter step-index core. The fiber is pumped by multimode pulses at $1061 \mathrm{~nm}$ and amplifies a signal at $1121 \mathrm{~nm}$. The total average-power gain reaches $9.2 \mathrm{~dB}$ with mode purity of $\sim 78.4 \%$. Without pumping ( $0 \mathrm{~dB}$ gain) the mode purity becomes $84.3 \%$. To the best of our knowledge, this is the first demonstration of stable amplification of an OAMM with such high charge in a large core fiber.

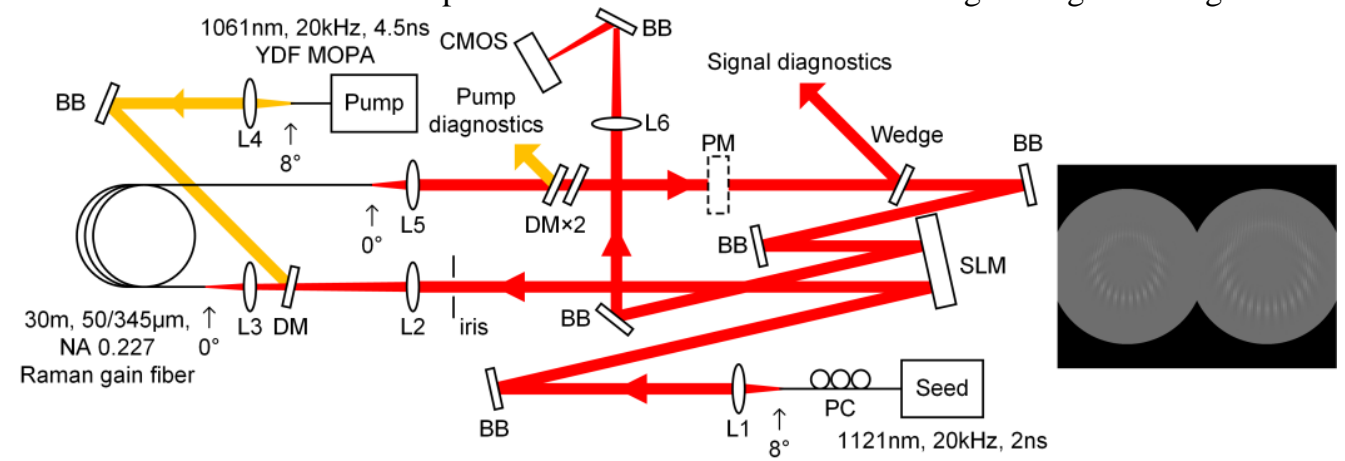

Fig. 1. Experimental setup of Raman amplification and mode purity measurement. PC - polarization controller; BB - broadband mirror; DM - dichroic mirror; PM - power meter. Inset shows the hologram displayed on the SLM.

Fig. 1 illustrates the experimental setup. This is similar to that of our previous work [2], but incorporates some modifications. The Raman seed now consists of a directly modulated 1121-nm FBG-stabilized diode laser (DL) and a Raman pre-amplifier (not shown) emitting 2-ns pulses at 20-kHz repetition rate. The seed beam is collimated by a plano-convex lens (L1, f=100 mm) and processed by a phase-only spatial light modulator (SLM, SLM-100, Santec) for conversion into the target OAMM. The SLM is partitioned into two equal halves to support both the generation and the decomposition through the use of optical correlation [4]. A fiber polarization controller orients the polarization of the incident seed beam to the preferred linear polarization of the SLM. A plano-convex lens (L2, $\mathrm{f}=200 \mathrm{~mm})$ and an aspheric lens (L3, $\mathrm{f}=8 \mathrm{~mm}$ ) are used to launch the generated OAMM into the Raman gain fiber. The fiber is pulled in-house from a commercial preform and has a pure-silica core of 50- $\mu \mathrm{m}$ diameter and 0.227 NA, with both ends perpendicularly cleaved to minimize mode distortion. The Raman pump pulses, coming from a MOPA (not shown) consisting of a modulated DL at $1061 \mathrm{~nm}$ and three amplifier stages, have a duration of $\sim 4.5 \mathrm{~ns}$ with $20 \mathrm{kHz}$ repetition rate. The pump pulses are combined with the signal pulse in a dichroic mirror (DM) and an aspheric lens. The launched pump at maximum gain has a peak power of $4.56 \mathrm{~kW}$ and pulse energy of $18.3 \mu \mathrm{J}$. The temporal overlap of the signal and pump pulses is tuned via their respective diode drivers. The output beam is collimated by an aspheric lens (L5, $\mathrm{f}=11 \mathrm{~mm}$ ) and the signal beam is extracted by two consecutive DMs. The diagnostics include a thermal power meter for the pump, a Ge power sensor for the signal, two $12.5 \mathrm{GHz}$ InGaAs detectors (ET-3500, EOT) connected to a 6$\mathrm{GHz}$ oscilloscope (Infiniium 54855A, Agilent) and an optical spectrum analyzer (AQ6317B, Ando). The transmitted signal beam is sent to the second half of the SLM and passes through a plano-convex lens (L6, $\mathrm{f}=750 \mathrm{~mm}$ ) for modal 
decomposition. The SLM is placed at the back focal plane of L6 and displays a series of holograms that encode the complex fields of OAMMs with neighboring charges for optical correlation with the incident beam [4]. A CMOS camera (DCC1545M, Thorlabs) placed at the focal plane of L6 captures the on-axis intensity of the first order diffraction from the SLM.

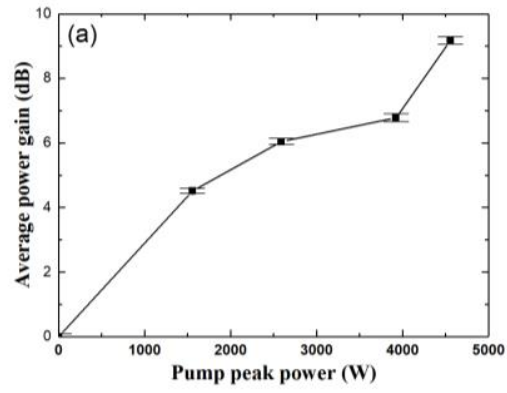

(d)

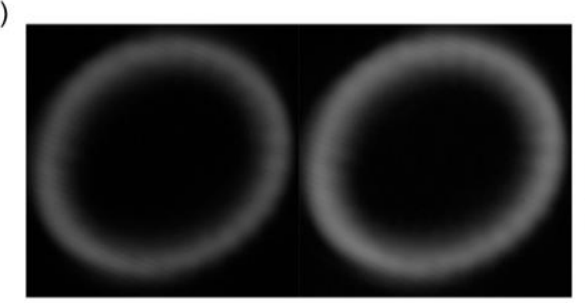

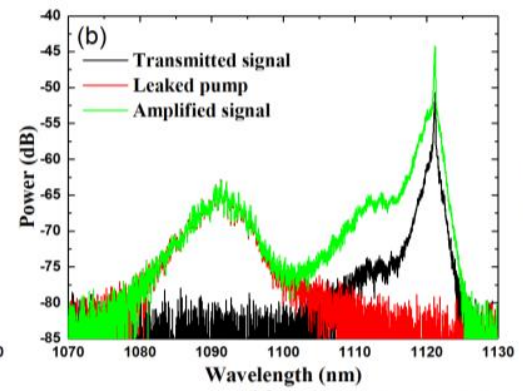
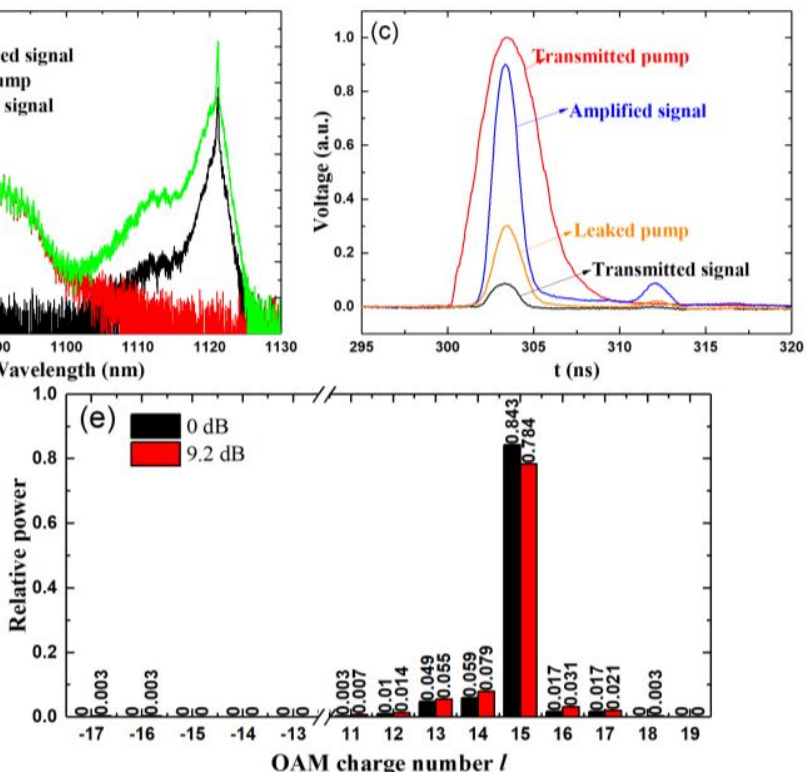

Fig. 2. (a) Measured average-power gain against launched pump peak power. (b) Optical spectra of the transmitted signal beam at $0 \mathrm{~dB}$ gain (black) and $9.2 \mathrm{~dB}$ gain (green). Red curve shows the spectrum of the leaked pump from the DMs. (c) Transmitted signal pulses without pumping (black), at $9.2 \mathrm{~dB}$ gain (blue) and leaked pump pulse (orange). Red curve shows the launched pump pulse in a different amplitude scale. (d) Far-field intensity profile of transmitted signal beam at $0 \mathrm{~dB}$ (left) and $9.2 \mathrm{~dB}$ gain (right). Brightness and contrast tuned for visibility. (e) Modal content of output signal at $0 \mathrm{~dB}$ (black) and $9.2 \mathrm{~dB}$ gain (red), calculated by normalizing the on-axis intensity to the total on-axis intensities of all listed modes.

The average power gain versus the launched pump peak power is shown in Fig. 2(a). The Raman gain is calculated as the ratio of measured average power of the transmitted signal with and without pumping after subtracting the pump power that leaks from the imperfect DMs, which is also shown in the spectra of transmitted beam in Fig. 2(b). At $9.2 \mathrm{~dB}$ gain, the transmitted signal reaches $5.41 \mathrm{~W}$ of peak power and $9.2 \mathrm{~nJ}$ of pulse energy after appropriate background subtraction. The pump launch is adjusted at every pump level for optimal spatial overlap between the multimode pump and donut-shaped signal beam. As a result, the gain enhancement is not linear with launched pump power. Fig. 2(c) shows the temporal traces of the pump and signal pulses. Fig. 2(d) illustrates the OAM mode profile without (left) and with the pump radiation (right). The elliptical mode shape is attributed to aberrations in our optical setup which are partially compensated during mode decomposition by tilting L6. The modal decomposition of the signal beam into the neighboring charges from +11 to +19 and conjugate charges from -17 to -13 are shown in Fig. 2(e). Here, we ignore the modal content of OAMMs with higher radial orders for simplicity and a more complete modal decomposition will be carried out later. A back-to-back measurement shows that the beam incident to the gain fiber has a purity of $\sim 98 \%$ for $l=15$. The transmitted signal without amplification has a mode purity of $84.3 \%$ and the amplified signal, after subtracting the leaked pump light, has a mode purity of $78.4 \%$. The weight of the target mode drops mainly because of increased weight of OAMMs with neighboring azimuthal orders, unlike the case of lower order OAMMs which couple primarily to the conjugate mode.

In conclusion, we have demonstrated stable propagation and Raman amplification of a single OAMM with $l=15$ in a large-core step-index fiber. Higher Raman gain is expected with longer gain fiber and a pump-signal frequency offset that is closer to the Raman gain peak.

This work is funded by AFOSR (FA9550-17-1-0007). Data presented here is available from University of Southampton at https://doi.org/10.5258/SOTON/D1163.

\section{References}

1. S. Zhu, S. Pidishety, Y. Feng, S. Hong, J. Demas, R. Sidharthan, S. Yoo, S. Ramachandran, B. Srinivasan, and J. Nilsson, Opt. Express 26, 23295 (2018).

2. S. Pidishety, S. Zhu, Y. Feng, B. Srinivasan, and J. Nilsson, Opt. Lett. 44, 1658 (2019).

3. P. Gregg, P. Kristensen, and S. Ramachandran, Optica 2, 267 (2015).

4. S. Pachava, A. Dixit, and B. Srinivasan, Opt. Express 27, 13182 (2019). 